\title{
Gordofobia, una lectura desde (y para) el Trabajo Social
}

\author{
Fatphobia, a Reading from (and for) Social Work
}

Gordofobia, uma leitura a partir do (e para o) Serviço Social

Ignacio Alonso Allende Allende*

\section{RESUMEN}

La gordofobia puede conceptualizarse, de modo general, como el miedo, rechazo e intolerancia a la gordura (propia o externa), la cual se refleja en distintas actitudes o comportamientos, siendo el principal de ellos la discriminación (directa o indirecta) hacia las corporalidades gordas. En este sentido, la premisa a desarrollar en el presente artículo será que la gordofobia es un fenómeno social problemático y que, por lo tanto, se levanta como un desafío para el Trabajo Social. De ahí que se buscará, en una primera instancia, comprender la gordofobia como una serie de lógicas internalizadas en la sociedad, desarrollando sus implicancias en un nivel psicosocial y contemplando la configuración de exclusión y violencia; en una segunda instancia, levantar una discusión que interpele al Trabajo Social a repensarse a sí mismo, evaluar sus propios patrones de intervención y considerar a la gordofobia como un fenómeno a intervenir.

\begin{abstract}
Fatphobia can be defined in general terms as the fear, rejection and intolerance to fatness (own or external), which is reflected in different attitudes or behaviors, the main one being (direct or indirect) discrimination towards fat people. In this way, the premise to be developed in this paper will be that fatness is a problematic social phenomenon and, therefore, a challenge for social work. From this starting point, it will attempt to understand fatphobia as a series of logics internalized within society,
\end{abstract}

Palabras clave: gordofobia, estigma gordo, intervención social, Trabajo Social.

Key words: Fatphobia, fat stigma, social intervention, social work.

Nacionalidad chilena, Estudiante de Trabajo Social, Universidad de Chile. E-mail: ignacio.allende@ug.uchile.cl 
with implications at the psychosocial level and leading to the configuration of exclusion and violence. This will be followed by a discussion that urges Social Work to rethink itself, evaluating its own intervention patterns and considering fatphobia as a phenomenon that must be addressed.

\section{RESUMO}

A gordofobia pode ser definida, de forma geral, como o medo, rejeição e intolerância à gordura (própria ou alheia), o que se reflete em diferentes atitudes ou comportamentos, sendo o principal a discriminação (direta ou indireta) em relação aos corpos de pessoas gordas. Neste sentido, a premissa a ser desenvolvida no presente artigo será que a gordofobia é um fenômeno social pro-

Palavras-chave: gordofobia, estigma da gordura, intervenção social, Serviço Social. blemático e que, portanto, surge como um desafio para o Serviço Social. Procura assim, em uma primeira instância, compreender a gordofobia como uma série de lógicas internalizadas na sociedade, cujas implicações são desenvolvidas em nível psicossocial e contemplando a configuração de exclusão e violência. Em segundo lugar, busca-se iniciar um debate que desafie o Serviço Social a repensar a si mesmo, a avaliar seus próprios padrões de intervenção e a considerar a gordofobia como um fenômeno que necessita ser abordado. 


\section{Introducción}

El neoliberalismo se ha configurado como uno de los modelos de desarrollo económico y social hegemónicos en el mundo contemporáneo. Este, de modo general, responde a una teoría de prácticas político-económicas, cuyos pilares son el fortalecimiento de los derechos de propiedad privada, los mercados libres y la libertad de comercio (Harvey, 2007). Sin embargo, su influencia no se limita solo al plano económico o a los funcionamientos del mercado, sino también a las formas en que se desarrolla lo social y se configuran las subjetividades de quienes lo construyen, configurándose como un discurso hegemónico que coloniza incluso las formas más naturales en que interpretamos, vivimos y entendemos el mundo, operando como una especie de ética en sí misma, capaz de guiar toda acción humana (Harvey, 2007). Sienta sus bases en la meritocracia, el individualismo y una justicia desde la desigualdad legítima (Basaure, 2015, citado en Castro-serrano, Borja y Flotts, 2018).

Particularmente, el cuerpo y las corporalidades han sido objeto de extensos debates, sobre todo en relación a cómo su construcción sociocultural ha variado, dadas las configuraciones histórico-contextuales a lo largo del tiempo. En esta línea, Mari Luz Esteban (2004), doctora en Antropología, expone que, en cualquier práctica cultural, el cuerpo está íntimamente ligado a lo social, dado que toda práctica social es, en cierto punto, una experiencia corporal. En esta línea, la construcción o connotación sociocultural del cuerpo no ha escapado al alcance de esta racionalidad neoliberal; en efecto, según algunos autores, las últimas décadas del siglo XX y comienzos del siglo XXI se han caracterizado por convertir al cuerpo en un símbolo de estatus, juventud, salud, energía y movilidad (Callinicos, 1993, citado en Esteban, 2004). De esta manera, el cuerpo se ha visto considerablemente instrumentalizado, cosificado y capitalizado, debido a la tendencia capitalista a mercantilizar todo orden de cosas, incluso los atributos humanos (Álvarez, 2017).

Foucault (2002), por su parte, en Vigilar y Castigar, sostenía que el cuerpo solo se considera fuerza útil cuando es, a la vez, un cuerpo productivo y sometido. En este sentido, expone que el cuerpo está inmerso en un campo político, un campo en el que:

las relaciones de poder operan sobre él (...); lo cercan, lo marcan, lo doman, lo someten a suplicio, lo fuerzan a unos trabajos, lo obligan 
a unas ceremonias (...). Este cerco político del cuerpo va unido, de acuerdo con unas relaciones complejas y recíprocas, a la utilización económica del cuerpo. (p. 32)

$\mathrm{Al}$ respecto, la ética neoliberal se ha encargado de informar y enfatizar la brecha entre cómo vivimos el cuerpo y cómo se supone que debemos disfrutar con él, levantando como exigencia estandarizada tener uno que represente esfuerzo y productividad (Harjunen, 2016), elementos que, por lo demás, connotan no solo el trabajo corporal y el ejercicio como un mérito y esfuerzo a reconocer, sino también a los cuerpos delgados como merecedores de validación y de lo exitoso, en planos no solo biologizantes sino también estético-culturales (Drinkwater, 2016). Dichas configuraciones y exigencias, sumadas a los saberes médicos, han posicionado a la delgadez de cuerpos fibrosos como lo hegemónico, lo ideal y lo deseable desde dimensiones no solo biológicas, sino también estéticas. En este sentido, la refinación e intervención del cuerpo (desde dietas rígidas e irresponsables para nuestro organismos hasta intervenciones quirúrgicas) se levantan como herramientas fundamentales para, no solo alcanzar el éxito corporal, sino también asegurar la productividad del cuerpo (Moreno, 2015; Drinkwater, 2016; Navajas-Pertegás, 2017; Esteban, 2004).

Así, la gordura empieza a configurarse como una contraparte o disonancia hacia lo configurado como hegemónico (recordemos, la delgadez). Asociándose a, entre otras, improductividad y descuido personal. Lo cual implica que, además de la patologización y del estigma sociocultural, se ha construido sobre las personas con cuerpos gordos ${ }^{2}$ la asociación de estas con lo indeseable y la proliferación de diversas formas de violencia que van desde lo simbólico hasta lo estructural, incluyendo formas de manifestación psicológicas e incluso físicas de discriminación. Dichas acciones se han conceptualizado desde el activismo gordo como "gordofobia".

Trasladando la discusión hacia un área disciplinar, parece imprescindible mencionar que el Trabajo Social tampoco ha sido la excepción

A lo largo del artículo utilizaré el concepto gordo/a y corporalidades gordas para referir a aquellas corporalidades con sobrepeso y obesidad, puesto que, se busca visibilizar, resignificar y desvergonzar la palabra gordo/a y ejercer resistencia contra la medicalización de la diversidad corporal. 
si de colonización por parte de la racionalidad neoliberal se trata. Los modos en que se desarrolla, la finalidad u horizonte que persigue y el carácter de su intervención, se han configurado, en su mayoría, como un medio de reproducción o naturalización de las lógicas de mercado y competencia imperantes, tanto en el mercado como en lo social. De esta manera, la mera idea de intervención ha contenido e implicado "una acción unilateral, externa y artificial, que se entromete en el curso de vida de personas y comunidades, identificando, desde una posición de superioridad moral, qué asuntos deben ser cambiados, prevenidos o erradicados en sus vidas cotidianas" (Muñoz, 2018, pp. 159-160), manifestándose, en su mayoría, como un acto de integración dirigido hacia quienes se han visto excluidos socialmente. Sin embargo, desde un ejercicio de resistencia frente a este modelo, podemos entender, desde un enfoque crítico, el Trabajo Social como aquel que posee su principio explicativo en la transformación social (Matus, 2018), es decir, como aquella disciplina que busca contribuir a la conformación de una sociedad en la que la justicia social sea legítima.

Es bajo esta última perspectiva que la falta de cuestionamiento a la naturalización de la violencia hacia las corporalidades gordas, desde la disciplina, se levanta como un déficit en lo que respecta al trabajo por alcanzar una sociedad en la cual la justicia social y el respeto por los cuerpos sea legítimo. De ahí que sea menester emprender un camino reflexivo y autocrítico frente a estas expresiones de violencia, y empezar a conceptualizar y reconocer la gordofobia como un fenómeno social problemático, contribuyendo a subsanar los vacíos en la discusión, tanto en Trabajo Social como en ciencias sociales. En este sentido, uno de los principales objetivos de este trabajo será levantar una crítica que interpele a la disciplina a problematizar la gordofobia como un desafío para su actuar disciplinar, considerando los impactos de sus lógicas al levantar intervenciones o participar de programas sociales, como también en términos de reconocerla como un fenómeno social que amerita intervención disciplinar.

\section{Gordofobia: opresión, estigma y fenómeno con impactos en lo psicosocial}

El origen de la palabra "gordofobia" viene del término "Fatphobia", puesto en circulación por un estudio realizado en 1984 por Robinson, 
Bacon y O'Reilly, en USA, para referirse a las actitudes negativas de la población local de Minnesota hacia los estereotipos que se relacionaban con las personas gordas (Álvarez, 2015). Sin embargo, el activismo gordo se ha apropiado del concepto, entendiéndolo como una opresión estructural. Al respecto, Magdalena Piñeyro (2016), activista gorda y fundadora de la plataforma "stop gordofobia", afirma que este fenómeno:

es un sistema que excluye y margina a la gente gorda de los ámbitos de la vida social (de la pareja, de los medios, del trabajo, del deporte, incluso de la calle). Y algo importante a tener en cuenta es que, de alguna $\mathrm{u}$ otra forma, TODAS LAS PERSONAS GORDAS SUFREN GORDOFOBIA (hombres, mujeres, trans...), por eso decimos que la gordofobia es una OPRESIÓN, algo estructural en nuestras sociedades.

Tomando esto en cuenta, en este artículo se conceptualizará la "gordofobia" como un sistema de opresión naturalizado en la sociedad, que consiste en la discriminación, intolerancia y rechazo a la gordura, ya sea propia o externa. Teniendo entre sus formas de manifestación elementos que van más allá del miedo a la gordura, tal es el caso de la burla, el chiste, los insultos, el acoso médico, la invisibilización del cuerpo gordo en los medios, entre otros. Por ello, se habla además de ésta como un conflicto cultural, social y político (Piñeyro, 2016; Álvarez, 2015).

Cabe destacar que, si bien la gordofobia afecta a todas las personas gordas, las dimensiones e impactos poseen diferencias al considerar otros sistemas de dominación. En esta línea, la reconocida activista Kelli Jean Drinkwater (2016) plantea que la gordofobia está profundamente arraigada en estructuras complejas, como el capitalismo, el patriarcado y el racismo. Sin embargo, a estas categorías podemos sumar la heterosexualidad y cisexualidad como regímenes políticos, y también el colonialismo occidental, en tanto han incidido en la configuración de exclusión social y los estándares de lo deseable. Estas apreciaciones son importantes al momento de tensionar la gordofobia, ya que "la transgresión encarnada de un cuerpo gordo es multidimensional, por lo que genera una amplia gama de reacciones y posturas" (Vargas, 2016, p. 75), y categorías como salud, género, sexualidad, capacidades, clase, piel, entre otras, incidirán directamente en la forma en que se expresará la violencia gordofóbica. 
En esta línea, cabe hacer mención a uno de los factores claves en la legitimación social de la superioridad moral de la delgadez y las dinámicas gordofóbicas: el discurso biomédico (Navajas-Petegás, 2017). Sin embargo, antes de profundizar en este discurso en particular, parece pertinente considerar que el discurso en general se entiende como aquel que "define y produce los objetos de nuestro conocimiento (...). También influencia cómo las ideas son puestas en práctica y usadas para regular la conducta de los otros mientras los sujetos son ubicados en relaciones de producción y de significación" (Foucault, 1994, p. 124). En este sentido, el discurso es un elemento que expresa los procesos de subjetivación de las personas y permite identificar las formas en que legitima el poder y el saber. Ahora bien, a pesar de que el discurso biomédico contra la gordura tiene su proliferación o surgimiento en la esfera de las disciplinas médicas, este se ha institucionalizado y arraigado en el ethos social colectivo.

Dicho discurso posee como principio explicativo la consideración de la gordura como peligrosa para la salud, lo cual vemos explícitamente en la definición de sobrepeso y obesidad que se levanta desde la Organización Mundial de la Salud (2018): "el sobrepeso y la obesidad se definen como una acumulación anormal o excesiva de grasa que puede ser perjudicial para la salud". Bajo esta noción las diferentes disciplinas médicas han articulado una serie de mecanismos y estrategias para moldear los cuerpos gordos insalubres hacia un estado de "normalidad" y salubridad, con dietas alimenticias favorables para la pérdida de peso, tratamientos farmacológicos y operaciones quirúrgicas invasivas.

$\mathrm{Al}$ analizar este discurso, vemos que la salubridad se configura como un dispositivo de control de los cuerpos, que apunta a adecuar aquellas corporalidades gordas e insalubres -y por ende anormaleshacia lo que discursivamente se ha construido hegemónicamente como normal -la delgadez-, configurando lo gordo (Moreno, 2015) como un indicador de lo anormal, de aquello que escapa de la norma, lo cual contribuye doblemente a la creación y reforzamiento del estigma que permite una asociación indiscriminada entre gordura, enfermedad, etiologías moralmente recriminables y rechazo (Herndon, 2014; Gard, 2011; Eller, 2010, citado en Vargas, 2016).

Cuando esta asociación de lo gordo y lo anormal se naturaliza en el ethos social, empieza a validarse toda forma de intervención que 
potencie o incite la transición hacia lo normal-delgado, desde tratamientos médicos hasta incluso la violencia, en tanto se configuran mecanismos que visibilizan la anormalidad de lo gordo e incitan a la adecuación hacia la hegemonía de lo delgado.

Esta asociación es uno de los impactos directos de la gordofobia en un nivel social, porque genera el estigma. Al respecto parece pertinente considerar los planteamientos de Goffman y Guinsberg (1970), quienes exponen que la sociedad construye medios para categorizar a las personas, identificando como "atributos" aquellas características que se perciben como normales, corrientes y naturales; de esta manera, el estigma referiría a esas características que cargan con una connotación negativa:

Mientras el extraño está presente ante nosotros, puede demostrar ser dueño de un atributo que lo vuelve diferente (...) y lo convierte en alguien menos apetecible (...). De ese modo, dejamos de verlo como una persona total y corriente para reducirlo a un ser inficionado y menospreciado. Un atributo de esa naturaleza es un estigma. (Goffman y Guinsberg, 1970, p. 12)

De esta manera, el estigma refiere a aquella categoría social que agrupa individuos que poseen características raras o que atentan contra la normalidad del statu quo. Siguiendo esta línea de análisis, la reiterada asociación entre gordura, anormalidad e insalubridad produce el "estigma de ser gordo" o "estigma gordo" (Friedman, 2012; Álvarez, 2015; Drinkwater, 2016; Navajas-Pertegás, 2017; Vargas, 2016).

Para profundizar en él, una primera observación es que no se trata únicamente de si estos cuerpos son considerados saludables o no, se trata un conflicto estético, cultural y político, que dirige la percepción de lo gordo hacia lo feo, lo insalubre, lo irresponsable, lo perezoso y lo moralmente sospechoso, estableciendo a la delgadez como la contraparte hermosa, responsable, exitosa y autocontrolada, posicionándola en un estatus de superioridad moral (Drinkwater, 2016) y haciéndola jugando un rol "determinante a la hora de (re)producir relaciones de poder y control, favoreciendo la desvalorización de algunos grupos a costa de otros que se sienten en algún aspecto superiores" (Bayer 2008, p. 465, citado en Navajas-Pertegás, 2017, p. 40). Por otro lado, parte del estigma gordo es posicionar en el sentido común la ingesta masiva de 
comida como la causa natural y lógica de la gordura, invisibilizando causas genéticas, hormonales o de alteración de procesos metabólicos.

Este estigma ha tenido diversas expresiones e impactos en la calidad de vida de las personas gordas, en términos de exclusión y discriminación, tal es el caso de la ridiculización y la burla, que se posicionan las dinámicas más comunes y naturalizadas para violentar y visibilizar la incomodidad con lo gordo. Estas dinámicas pueden contener una doble intención: provocar un sentimiento de vergüenza en la víctima, por existir dentro de un cuerpo "anormal", e incitarle a adecuarse a los parámetros y estándares normales mediante la culpa y la presión. Álvarez (2015), activista gorda, vegana y lesbiana, se refiere a la burla como una forma de control impresionante en la que, quien se burla, adquiere el poder de deslegitimar el cuerpo de otro, añadiendo que "las burlas y la discriminación se justifican como un problema individual, reflejado en el cuerpo gordo; y si una se opone o se molesta por algún comentario, apelan a que es por tu bien, por tu salud, para ayudarnos" (Álvarez, 2015, pp. 40-41).

De esta manera, se generan una serie de impactos en la subjetividad de las personas gordas, uno de ellos refiere a la internalización de todos estos patrones y lógicas gordofóbicas y su aplicación constante hacia sí mismas. Esto se ha enunciado desde el activismo como "gordofobia internalizada", esto, sumado a la constante presión del medio sociocultural, puede llevar a trastornos emocionales y psicofisiológicos (Friedman, 2012).

En el primer caso, puede relacionarse meramente a cuestiones psicoemocionales, como la frustración y la baja autoestima, dado el incumplimiento de expectativas sociales de belleza (Vargas, 2016), la constante exclusión por parte del entorno social y ser el objeto de burlas y violencia de carácter simbólico. En el segundo, a la proliferación de ansiedad y/o depresión, e incluso a la configuración de un ambiente de alto riesgo para la detonación de trastornos de la conducta alimentaria ${ }^{3}$. Recordemos que dichos trastornos son producidos multifactorialmente y son

En adelante TCA. 
afectados por el ideal de belleza que propugnan los medios de comunicación de extrema delgadez, una idea de belleza representada por modelos, mujeres y hombres cuyas proporciones, peso y estatura son una auténtica excepción estadística (...). Ideales que muestran la delgadez como símbolo de independencia, éxito social, personal y profesional. (Steiner H, Lock J., citado en Vargas-Baldares, 2013, pp. 476-477)

En este sentido, estas mismas exigencias socioculturales pueden cultivar dismorfia corporal miedo excesivo a engordar, entre otros. Los cuales, a su vez, pueden ser considerados detonantes y/o síntomas de TCA más complejos, como atracones (Binge eating disorder) anorexia y bulimia (Portela de Santana et al., 2012; Vargas-Baldares, 2013; Associació contra l'Anorèxia i la Bulimia, 2014; National eating disorders, 2016). De hecho, son varias las fuentes de estudio biopsicosocial que plantean que, elementos como la promoción cultural del ideal de la delgadez, los estigmas o prejuicios sobre el peso y el tamaño, la insatisfacción corporal, la presión social y sus expresiones discriminantes (elementos que por lo demás podrían entenderse como expresiones a escala microsocial de la gordofobia y también como impactos de internalización de sus lógicas) se constituyen como factores de riesgo en el desarrollo de los TCA (Véase: Portela de Santana et al, 2012; Associació contra l'Anorèxia i la Bulimia, 2014; National eating disorders, 2016).

Al considerar lo anterior, la gordofobia y sus implicancias psicosociales, tanto en términos de discriminación gordofóbica como en interiorización y cultivo de detonantes de TCA, se puede ya visibilizar como esta se configura como un fenómeno social problemático, en tanto atenta contra el bienestar, libertad e integridad de las personas con corporalidades gordas.

Adicionalmente, dicho recorrido permite visibilizar la naturalización de la violencia gordofóbica en nuestras dinámicas de interacción social y entender que sus lógicas y discursos han calado en lo profundo de la dinámica social, configurándose en una especie de lo que ya Foucault (2008) denominaba como "regímenes de la verdad". Estos se caracterizan por sostener una "política general" de la verdad, en la que se acogen y hacen funcionar ciertos tipos de discursos como verdaderos o falsos, y se sanciona a otros (Foucault, 2008). Adicionalmente, 
este autor plantea que la economía política de la verdad está ligada a cinco rasgos importantes:

La «verdad» está centrada sobre la forma del discurso científico y sobre las instituciones que lo producen; está sometida a una constante incitación económica y política (necesidad de verdad tanto para la producción económica como para el poder político); es objeto, bajo diversas formas, de una inmensa difusión y consumo (circula en aparatos de educación o de información cuya extensión es relativamente amplia en el cuerpo social, a pesar de algunas limitaciones estrictas); es producida y transmitida bajo el control no exclusivo pero dominante de algunos grandes aparatos políticos o económicos (universidad, ejército, escritura, media); finalmente, es el envite de todo un debate político y de todo un enfrentamiento social (luchas «ideológicas». ( Foucault, 2008, pp. 143-144)

De esta forma, en la gordofobia se identifica una serie de dispositivos de legitimación (el discurso biomédico, la construcción del estigma "gordo", la necesidad neoliberal de cuerpos productivos, etc.) que potencian la construcción de una verdad hegemónica que posiciona a la gordura y los cuerpos gordos como merecedores de rechazo, segregación y discriminación. Al reconocer estos factores, tomar un posicionamiento antigordofóbico y hablar sobre cómo contribuir a una sociedad con legítima justicia social, libertad y reconocimiento para la totalidad de las personas, la mera existencia de la violencia (en sus diferentes espectros y formas) hacia las corporalidades gordas y su escasa discusión es una interpelación directa al Trabajo Social para repensarse a sí mismo como disciplina transformadora.

\section{Trabajo social y gordofobia}

Las violencias hacia las corporalidades gordas es un fenómeno que se encuentra poco teorizado, problematizado e investigado por el Trabajo Social (Mik-Meyer, 2010; Fridman, 2012, Návajas-Petergas, 2017) en el mundo y aún menos el Latinoamérica. Por ello, existe un desconocimiento en cuanto a la magnitud de su impacto social y acerca de "la profundidad de los efectos causados por los sesgos sobre la gordura que mantienen las/os trabajadoras/es sociales con sus usuarias/os, y los sesgos del alumnado de trabajo social" (NavejasPetegás, 2017, p. 41). 
Esto se presenta como un déficit necesario de subsanar; pues hacerlo no solo implicaría la visibilización de este fenómeno sino que también se avanzaría en la deconstrucción del discurso gordofóbico $y$, con ello, en el cuestionamiento de la verdad totalizante y estigmatizante que prolifera desde él. También, pondría en disputa la verdad e institucionalización de dinámicas socioculturales que oprimen y violentan las subjetividades de las personas gordas, lo cual, a su vez, contribuiría con la articulación de formas imaginativas y creativas de resistencia positivas a la dominación (Yeatman, 1994, citado en Matus, 2017).

Ahora bien, a pesar de la baja teorización e investigación presente en este campo, son diversas las autoras que, al incursionar en la vinculación entre gordofobia y Trabajo Social, afirman que este último, dado su accionar disciplinar en el área de la salud y la sanidad, se ha visto altamente involucrado como afianzador y reproductor de las premisas del discursos biomédico contra la gordura (May Fridman, 2012; Navejas-Petás, 2017), sobre todo al considerar que "el ámbito sanitario y el saber popular contemporáneo consideran la gordura como consecuencia de una ingesta excesiva de alimentos" (Santiso, 2001, citado en Navejas-Petás, 2017, p. 39). Al respecto, May Friedman (2012) afirma que, frecuentemente, los trabajadores sociales que se desempeñan en contextos relacionados con la famosa "guerra contra la obesidad" (o escenarios que involucren directamente la relación con gente que posee cuerpos gordos) desempeñan un rol de ayuda y asistencia psicoemocional, con el fin de que estas personas superen sus problemas y trabas emocionales, puedan generar un cambio de hábitos y comenzar la anhelada trayectoria para perder peso y "encajar" en los cánones normativos establecidos como hegemónicos. Afirmando, además, que son partícipes y masificadores en cuanto a "entregar el mensaje de que los cuerpos gordos sólo merecen atención si están en transición [a la delgadez]" (Friedman, 2012, p. 63).

De esta manera, las implicancias de reproducir lógicas gordofóbicas en el actuar disciplinar tendrían un impacto opresivo en lo que respecta a cómo las subjetividades de las corporalidades gordas se perciben a sí mismas, puesto que naturaliza y legitima aún más la relegación de lo gordo al lenguaje y espacio de la vergüenza. En este sentido, Friedman (2012) expone que: 
Si bien estas intervenciones son indudablemente bien intencionadas y pueden ser, por una variedad de razones, dirigidas por el usuario son, sin embargo, profundamente problemáticas. (...) las intervenciones de trabajo social que se centran en la pérdida de peso ayudan a mantener los estigmas asociados con la grasa, así como también aumentan el estigma interiorizado y la responsabilidad individualizada de la opresión de la grasa. (p. 63)

Así se despliega un nudo crítico a tensionar, en tanto existe una especie de reducción del conflicto que experimentan estas personas a su mero estado corporal y búsqueda de cambio, invisibilizando y desconociendo la problemática de exclusión sociocultural y los impactos psicoemocionales que ellas experimentan las personas gordas por el mero hecho de poseer cuerpos disidentes ${ }^{4}$; y no solo eso, sino también los detonantes psicosociales de la gordura, que van más allá de solo ingerir más calorías de las necesarias. Cabe destacar que este vaciamiento de contenido de problemáticas sociales, característico del neoliberalismo, pone énfasis en el descontrol, los problemas de autoestima, la estabilidad emocional, entre otros, para culminar en la culpabilización de las y los sujetos respecto de su propia situación de exclusión y anormalidad (Navejas-Petegás, 2017).

En este sentido, el biopoder juega un papel fundamental respecto del control de los cuerpos y como matriz de análisis para el presente escrito. Antes, sin embargo, parece pertinente indagar en los conceptos de "anatomopoder" o "anatomopolítica" y el de "biopolítica". La anatomopolítica se refiere a las formas de educar y disciplinar el cuerpo individual, de volverlo un cuerpo útil y dócil (Foucault, 1998c, citado en Sossa-Rojas, 2011). De esta manera, refiere a una mecánica del poder que "define cómo se puede hacer presa en el cuerpo de los demás, no simplemente para que ellos hagan lo que se desea, sino para que operen como se quiere, con las técnicas, según la rapidez y la eficacia que se determina" (Foucault, 2002, p. 142).

La biopolítica, por otra parte, para Michel Foucault (2001), no solo se relaciona con los individuos y las formas en que se condicionan sus cuerpos y desarrollo natural (natalidad, mortalidad, fertilidad, desa-

Cuerpos fuera de la norma hegemónica 
rrollo, etc.), sino también con lo que respecta a la población y los modos de gobierno colectivo, teniendo en cuenta las acciones, discursos y saberes que modifican los comportamientos, y la manera en que estos se desarrollan con el entorno. Entonces, "la biopolítica tiene que ver con la población, y ésta como problema político, como problema a la vez científico y político, como problema biológico y problema de poder" (Foucault, 2001, p. 222).

Estos dos conjuntos (anatomopolítica y biopolítica) conforman lo que se denomina "biopoder". Al respecto, Sossa (2011) expone que "aquí, ambos conceptos operan medularmente para producir productividad, en el primer caso: en una persona, en el segundo: en toda la sociedad" (p. 18). De esta forma, el biopoder es una noción que fluctúa entre la microfísica de las estrategias y la macrofísica de las tecnologías de poder o dispositivos de control (Toscano, 2008).

Ahora bien, al considerar el biopoder como una matriz analitica y, a su vez, esta premisa de la reducción del conflicto gordo a la ingesta de alimentos y riesgo cardiovascular, parece pertinente mencionar que, en materia de política e intervención pública nacional chilena, existe el "Plan Nacional de prevención del sobrepeso y la obesidad en niños, niñas y adolescentes", y la "Política Nacional de Alimentación y Nutrición", los cuales poseen, como elemento común, en distintos niveles, apuntar a un fortalecimiento de dispositivos focalizados en la regulación de la ingesta alimenticia, persiguiendo cultivar no solo hábitos alimentarios "saludables", sino también de conducta y trabajo corporal, en cuanto a la realización de ejercicios aeróbicos, entre otros, con el fin de reducir los niveles de gordura en la población chilena. De esta manera, se pone principal atención a la regulación de la conducta alimentaria de los individuos, lo cual refleja que el Estado está esperando de sus ciudadanos y ciudadanas personas con capacidades físicas y aeróbicas efectivas, propósito que podríamos relacionar con la premisa neoliberal de entender el cuerpo como mercancía y un medio para efectivizar y medir la productividad, sujeto más valioso y responsable en tanto cumple con los requisitos y expectativas de los estándares y saberes hegemónicos.

Dichos planes presentan estructuración simplista y cargada de lógicas biomédicas que separan las dimensiones biopsicosociales de las personas. Por ejemplo, la atención psicológica y la nutricional se plantean de manera paralela, pero no juntas, sino que se disocian, buscan- 
do cada una, desde su particular contribución, el cambio ansiado: un estado de productividad corporal eficiente y saludable. Cuando, al menos desde una perspectiva crítica, debería realizarse desde un punto de concentración simultáneo, que parta desde la aceptación corporal para el cambio consciente y no forzado por el punitivismo, miedo o el riesgo biológico que, se ha expuesto, encarna lo gordo. Adicionalmente, estos planes de prevención e intervención en gordura no realizan un fuerte acompañamiento psicosocial en torno a las causas que detonan la gordura, más allá de la ingesta de alimento o el sedentarismo; ni mucho menos despliegan un proceso de concientización sobre las diversas violencias que sufren los cuerpos gordos y cómo estas pueden incidir en la potenciación de trastornos alimentarios, tal y como expusimos.

Cabe destacar que esta reducción no solo se expresa en esta lógica de riesgo y control de los cuerpos, sino también en el uso del lenguaje. Un ejemplo de ello es el caso del programa de intervención social chileno "Programa Vida Sana", que busca combatir la gordura dado el riesgo cardiovascular, y que incluye, dentro de sus objetivos específicos, "Mejorar en niños y niñas entre 6 y 19 años la relación Z Score de IMC y Perímetro de Cintura. [y] Mejorar la condición física en términos de capacidad muscular y aeróbica" (PVS, 2012, p. 8). Si bien se entiende que los objetivos del programa son potenciar la calidad de vida y salir de una situación de riesgo cardiovascular, lo problemático, desde un análisis crítico, es cómo, por un lado, se construye un discurso identificando a personas gordas como focos de intervención insalubres y fuera de la norma, y, simultáneamente, se les categoriza como sujetos y sujetas incapaces de movilizarse de manera autónoma por su propio bienestar integral, reproduciendo la presunción de una relación directa entre gordura y falta de capacidad muscular y aeróbica, cuando asumir que un cuerpo gordo está enfermo y falto de trabajo corporal/ muscular, mientras que uno delgado está saludable y resistente es un razonamiento propio de la gordofobia ${ }^{5}$.

$\mathrm{Al}$ respecto, deconstruir este régimen de la verdad totalizante y desplegar un raciocinio que entienda que tanto personas con cuerpos delgados como gordos pueden tener "malos/buenos" hábitos alimenticios y "buena/mala" resistencia cardiovascular, es un avance mínimo que

Al respecto, véase; fat studies / fat critical studies. 
visibiliza una ruptura contra el discurso gordofóbico, en tanto ambos cuerpos pueden acumular otros factores de riesgo más allá del peso, tal es el caso del colesterol, déficit de masa muscular, procesos metabólicos alterados, entre otros. En este sentido, la crítica no va en la dirección de menospreciar o rechazar el trabajo por la difusión de hábitos que favorecen un "buen" estado corporal o de salud, sino en términos de cómo se reduce el conflicto de la gordura al mero estado biológico/corporal, no contemplando una dimensión integral que contemple sus esferas psicosociales.

De esta manera, ¿cuántos programas o intervenciones en esta área son realizados con un enfoque de nutrición, en el que se celebre la diversidad corporal eliminando estigmas y juicios sustentados en falacias sobre los diferentes estados corporales? Y, a su vez, ¿cuántos que sean enfáticos en la promoción de la idea de que la salud es posible en todas las corporalidades? ${ }^{6}$. O, por otro lado, ¿ cuántos de los numerosos programas de prevención e intervención en gordura con niños, niñas y adolescentes incluye acompañamiento psicosocial constante, no solo para que los cambios de conducta alimentaria no sean invasivos sino también para evaluar que la motivación de cambio de hábitos no encarne odio hacia el cuerpo gordo? La respuesta parece alarmante, pues, como veníamos problematizando, en los diferentes dispositivos existe una preocupación por la reducción de los índices de gordura, sobrepeso y obesidad, considerando factores o detonantes sociales contextuales, sin atender a los impactos psicoemocionales de cargar con el estigma y la opresión social de encarnar un cuerpo gordo, la exclusión de aquellos espacios que, desde el discurso hegemónico, no son propios de estos, a los impactos de empezar un proceso de transición de hábitos y corporalidad desde lo disidente a lo hegemónico y al riesgo de reforzar lógicas de (auto)percepción gordofóbicas.

De ahí la necesidad de problematizar la gordofobia desde la disciplina y de entenderla como un fenómeno social problemático. Reconocer y tomar un posicionamiento es un primer desafío para el Traba-

\footnotetext{
6 "Este tipo de paradigma, desde diferentes movimientos críticos de la Nutrición y Dietética, se ha conceptualizado como nutrición incluyente, y refiere a una apuesta innovadora donde la nutrición se levanta ya no desde una lógica reproductora de estigmas, sino desde la aceptación corporal y la promoción de salud de maneras no excluyentes y sin reproducir opresiones y violencias socioculturales en los cuerpos"
} 
jo Social contemporáneo, y ello tiene una implicancia contradictoria, ya que se reconoce su potencial de transformación, pero también su potencial gordofóbico, en tanto puede reproducir discursos opresivos contra las personas gordas. En este sentido, es menester ejercer un juicio crítico acerca de cómo estamos desarrollando la acción disciplinar y el lenguaje con el que construimos los diferentes procesos de intervención social en el espacio de la gordura. Por otro lado, un segundo desafío consiste en reconocer este fenómeno como un terreno de intervención, es decir, considerarlo como un fenómeno social, ante el cual se hace urgente levantar el accionar disciplinar y desplegar intervenciones capaces de menguar su hegemonía y nivel de impacto, igualmente como se hace con otros fenómenos sociales problemáticos.

\section{La gordofobia como terreno de intervención e investigación}

Tomando como lente analítico un Trabajo Social crítico, que entiende la intervención social "como una forma de abordar un fenómeno social que ha sido conceptualizado como problemático y que [...] requiere ser intervenido para cambiarlo, mejorarlo, prevenirlo o erradicarlo" (Muñoz, 2018, p. 159), pensar en un actuar disciplinar que considere a la gordofobia como un fenómeno sobre el cual intervenir en pos de su erradicación parece un desafío contingente, que nos invita a pensar la gordofobia no solo como un terreno de intervención, sino también, como ya se venía exponiendo, de investigación.

Respecto de este ámbito, parece pertinente destacar el campo de los estudios de la gordura (Fat Studies) y el desafío inherente de la involucración disciplinar. En el mundo, la violencia gordofóbica es un tema de investigación "en pañales", que poco a poco se ha ido configurando como una línea de investigación. En países norteamericanos, diversos estudios ponen especial énfasis en la opresión a las personas que poseen cuerpos gordos y la deconstrucción y desmitificación de ciertos discursos en contra de estos.

Los denominados "Fat Studies", o también "Critical Fat Studies", refieren, según Marilyn Wann (2009), a un campo radical que va directo a problematizar los sistemas de creencias relacionados con el peso y el estado corporal. Adicionalmente, dicha autora plantea que los Fat studies dejan de ser lo que son cuando se asume, por ejemplo, que las 
personas gordas deben dejar de serlo, que estas no pueden disfrutar de buena salud y que estéticamente lo gordo es propiamente feo y lo delgado precioso. En este sentido, esta corriente no solo se opone a la reducción de la diversidad del peso humano, sino que pone en disputa lo que las personas y sociedades construyen respecto de la realidad y las dinámicas psicosociales y mitificaciones en torno al peso, exponiendo la premisa de que la salubridad es posible en todas las tallas (Wann, 2009).

Deborah Lupton (2013), en una recopilación y exposición de los principales hallazgos expuestos por investigadoras e investigadores de esta línea, principalmente en materia biomédica, afirma que "la gordura es a menudo un síntoma más que la causa de la mala salud y la enfermedad" (Lupton, 2013, p. 3). Adicionalmente, recuerda que no hay evidencia estadística significativa para posicionar a la gordura como un equivalente a un riesgo de mala salud, salvo en los caso de gordura hiperextrema o en lo que se denomina, desde las disciplinas médicas, "obesidad mórbida”, y que, en general, un mayor peso puede ser incluso protector de la salud en, por ejemplo, personas mayores (Campos, 2004; Gard y Wright, 2005; Gard, 2005, citado en Lupton, 2013). Junto con eso, se hace énfasis en que la literatura e investigación epidemiológica no ha podido demostrar que:

una pérdida de peso significativa mejore el estado de salud de las personas gordas. De hecho, los continuos intentos de las personas gordas por perder peso pueden ser en realidad negativos para su estado de salud, si implican dietas severas, estar atrapados en un ciclo de pérdida y aumento de peso o pobres hábitos alimenticio. (Lupton, 2013, p. 3)

Estos hallazgos son contribuciones del área investigativa de los Fat Studies a la indagación respecto de la desmitificación y deconstrucción del discurso biomédico. Por otra parte, ahondando en las dinámicas de lo social y la opresión gordofóbica naturalizada en el contexto contemporáneo, se han realizado investigaciones visibilizando la relación entre gordura y género: la construcción de la estética y los imaginarios corporales de lo femenino, lo masculino y lo disidente (Rothblum \& Solovay, 2009); discriminación a personas gordas en la atenciòn primaria de salud (Návajas-Petergas, 2017; Fridman, 2012), e incluso respecto de lógicas gordofóbicas e infancia. Con relación a esto último, 
se expone que las personas experimentan miedo a engordar desde los cinco años y que niños y niñas con cuerpos gordos tienen más probabilidad de intentar suicidarse que los delgados (Davison y Birch, 2001; Libbey, Story, Neumark-Sztainer y Boutelle, 2008, citado en NavajasPetergás, 2017), y que, de hecho, poseer una corporalidad gorda te hace un blanco fácil y común para los acosadores/bullyes. En esta línea, la literatura indica que las formas de discriminación hacia los cuerpos gordos en niños, niñas y adolescentes (NNA) no solo son comunes sino también aceptadas (Weinstock \& Krehbiel, 2009). Hago mención a este ámbito no solo para visibilizar la naturalización del acoso y violencia gordofóbica desde la infancia y algunos de sus impactos psicoemocionales, sino también para dejar constancia de cómo estos son también una alerta e interpelación directa para profundizar en la investigación como fenómeno social problemático, dadas las posibles teorizaciones o hipótesis que pueden generarse, no solo sobre su escala, impacto, alcance y evolución, sino también sobre su intervención.

Volviendo a lo que nos convoca, a pesar de la considerable evolución y construcción de esta área investigativa, cabe volver a enfatizar que, desde el Trabajo Social latinoamericano, esta temática ha sido poco abordada. Este vacío teórico es alarmante: debemos reconocer primeramente en nuestra disciplina las dinámicas gordofóbicas y avanzar en la indagación de, por ejemplo, la construcción de lo (in) deseable corporalmente, las trayectorias de las personas gordas, las experiencias de violencia y sus dimensiones, considerando las diferentes categorías de opresión y/o privilegio que proliferan desde otros fenómenos sociales (como es el colonialismo, el sexismo, la cisheteronorma y el género), y cómo este fenómeno ha podido incidir, tanto en el desarrollo interpersonal como también en la autopercepción de las personas gordas. Estas tareas son fundamentales para generar un posicionamiento disciplinar crítico respecto de este fenómeno, y posibilitar un avance en términos teórico-prácticos que permita desplegar intervenciones con una episteme antigordofóbica que haga frente a los escenarios de violencia ya mencionados.

En una dirección distinta, desde un enfoque crítico que considere los aportes epistemológicos de un enfoque postestructuralista al Trabajo Social, se buscará desplegar propuestas o enfoques de cambio social endogámicos, pragmáticos y sensibles al contexto sociohistórico, 
que busquen generar la emancipación de formas de opresión y relaciones de poder asimétricas, pero que también exijan que, quienes tomen el rol de activistas políticos, se posicionen desde una actitud críticamente autorreflexiva respecto de sus ideales emancipadores (Healy, 2001, citada en Matus, 2017). En este sentido, algunas estrategias para generar intervención directa, son la concientización y la incentivación $\mathrm{u}$ apoyo para el desarrollo de identificaciones colectivas o construcción de un sujeto social que se organice frente a este fenómeno.

La concientización apunta a un proceso de reflexión crítica en el que, quienes se ven oprimidos, pasan desde una posición de autoinculpación a la comprensión de los orígenes estructurales de sus padecimientos (Finn, 1994; Gutiérrez, 1995, citado en Healy, 2001). La concientización es un proceso que "no se limita a la reflexión racional, sino que se extiende, en último término, a un cambio fundamental, tanto de las relaciones personales en las que intervenga cada cual como en los contextos sociales más amplios" (Healy, 2001, p. 53). Aquí la articulación de trabajadores sociales, en conjunto con movimientos activistas gordos, podría servir para llevar a cabo programas o intervenciones sociales que faciliten la deconstrucción del estigma y la resignificación del cuerpo gordo, entendiendo que carga con una opresión histórica. La concientización juega puede llevar consigo la "redenominación" de la experiencia, de manera que se hagan explícitas las dimensiones políticas de la propia experiencia individual de desventajas o sufrimientos (Van Den Bercgch y Cooper, 1986; Dominelli y MCleod, 1989, citado en Healy, 2001, p. 53). De esta manera una adecuada reflexión y concientización frente al fenómeno permitiría generar una visibilización exhaustiva de las formas de violencia a las que se ven sometidas las corporalidades gordas y, a la vez, una reflexión crítica acerca del considerable impacto de la reproducción, no solo de la violencia, sino también del lenguaje gordofóbico.

Siguiendo estas premisas, la segunda proposición responde a acompañar la identificación colectiva y el reconocimiento del impacto social de la gordofobia. Esto apunta a estimular la organización y apoyar los procesos de creación y funcionamiento de colectivos o proyectos que busquen no solo tensionar la gordofobia, sino también levantar instancias de contención, reparación y resistencia. En este sentido, el Trabajo Social no debe tomar una posición de protagonismo, sino, 
todo lo contrario, de catalización e incentivación a la organización colectiva, o activismo político, de quienes se ven propiamente en situaciones de violencia gordofóbica, y acercar sus demandas hacia la institucionalización burocrática (Healy, 2001). De esta manera, conseguir que se conceptualice en el discurso pùblico el desafío de entender la gordofobia como un fenómeno problemático que debe erradicarse. Sin embargo, parece preciso mencionar que estas estrategias son solo un pequeño espectro de posibilidades respecto del potencial de acción de la disciplina frente a este fenómeno, ya que, sin duda, queda mucho camino reflexivo y propositivo al respecto.

\section{Reflexiones finales}

A lo largo del ensayo se ha desglosado y expuesto críticamente el fenómeno gordofobico presente en la sociedad contemporánea, y visibilizados sus respectivos impactos en la trayectoria y subjetividad de las personas con cuerpos gordos. Asimismo, se ha expuesto cómo el cuerpo, bajo el paradigma neoliberal, se presenta como un elemento que representa, entre otras cosas, productividad, capitalización, cultura, estética e identidad (Esteban, 2004; Álvarez, 2015, 2018; Vargas, 2016; Drinkwater, 2016). Así, también, se ha conceptualizado la "gordofobia" como una serie de prácticas y discursos que la revelan en sí misma como un fenómeno social problemático, cuya mera existencia y constante reproducción se presentan como un obstáculo considerable para el desarrollo de una sociedad en la cual la justicia social, la libertad plena y, obviamente, el reconocimiento de la dignidad y pluralidad inherente a los cuerpo sean legítimos.

Considerando esto, y particularmente que las diversas formas de opresión que proliferan desde el fenómeno han colonizado áreas que van desde la salud hasta lo estético, desde lo político hasta lo cultural, la gordofobia parece urgente de problematizar desde el Trabajo Social, en tanto este se entienda como una disciplina que, desde un enfoque crítico, persiga la transformación social (Matus, 2018) en pos de la emancipación de las personas de las diferentes formas de exclusión y marginalización característicos de la sociedad contemporánea. Es urgente abrir espacios de discusión, tanto en la práctica, en la academia como en la sociedad, para visibilizar y reconocer la gordofobia como un fenómeno social problemático y opresivo. 
Asimismo, parece urgente repensar el Trabajo Social desde una episteme postestructuralista, que piensa el camino a la emancipación desde la creación de condiciones adecuadas para el diálogo, la controversia y el alzamiento de las voces de quienes están padeciendo los impactos directos de la opresión estructural (Matus, 2017). Es un desafío disciplinar levantar y poscionarse desde un Trabajo Social antigordofóbico, que construya modelos de intervención dirigidos a tensionar y contribuir a la erradicación de la gordofobia como fenómeno problemático, y caminar hacia una transformación social basada en la equidad y la justicia social.

En este sentido, se debe reflexionar acerca del Trabajo Social como potencial reproductor de gordofobia y afianzador pasivo de su alcance, pero también como potencial interventor e investigador. En necesario abrir espacios de discusión y teorización para reconocer las formas de discriminación y exclusión que, desde la gordofobia (y su relación con otros sistemas de opresión), proliferan, dotando a la disciplina de una marco analítico que posibilite desplegar una acción disciplinar y nos acerque a una sociedad más justa, en la que categorías o características propias no produzcan un desequilibrio o asimetría en las relaciones de poder y el cuerpo no sea más que un cuerpo.

\section{Bibliografía}

Álvarez, S. (2018) Capitalismo neoliberal y cuerpo. Papeles de relaciones sociales y cambio global, 137. Primavera 2017. Recuperado de: https://www.fuhem.es/wp-content/uploads/2018/12/ papeles_137.pdf

Álvarez, C. (2015). La cerda punk: ensayos desde un feminismo gorda, lésbiko, antikapitalista \& antiespecista. Recuperado de: https://www.bibliotecafragmentada.org/wp-content/ uploads/2014/10/La_cerda_punk.pdf

Associació contra L'anorèxia i la Bulímia. (2014). Factores de riesgo para sufrir un TCA. Recuperado de: http://www.acab.org/ es/que-son-los-trastornos-de-la-conducta-alimentaria/factores-de-riesgo-para-sufrir-un-tca

Castro-Serrano, B. y Flotts, M. (2018). ¿Transformación social sin cambio? Puntualizaciones para un nuevo imaginario del trabajo social. En: M. Flotts y B. Castro (Eds.), Imaginarios de transformación: el trabajo social revisitado (pp. 21-46). Santiago de Chile: RIL Editores. 
Drinkwater, K. (2016). The Fear of Fat - The Real Elephant in the Room. Recuperado de: https://tedxsydney.com/talk/the-fear-offat-the-real-elephant-in-the-room-kelli-jean-drinkwater/

Esteban, M. L. (2004). Antropología del cuerpo. Género, itinerarios corporales, identidad y cambio. Barcelona: Bellaterra.

Espinoza, R. (2013) Estrategias de intervención en obesidad de niños, adolescentes y adultos. Actividad Física, Nutrición y Psicología. Recuperado de: http://wwwl.paho.org/chi/images/ PDFs/12.\%20Padre\%20Hurtado\%20Progranma\%20Vida\%20 sana\%20intervenci\%C3\%B3n\%20en\%20obesidad\%20en\%20 ni\%C3\%B1os\%20y\%20adolescentes_Rodrigo\%20Espinoza.pdf

Friedman, M. (2012). Fat is a Social Work Issue: Fat bodies, moral regulation, and the history of social work. Intersectionalities: A Global Journal of Social Work Analysis, Research, Polity, and Practice, 1, 53-69.

Foucault, M. (1994). Power: the essential works of Michel Foucault 19541984. London: Penguin.

Foucault, M. (1998) Historia de la sexualidad. México: Editorial Siglo Veintiuno.

Foucault, M. (2001). Defender la sociedad. Curso en el Collège de France (1975-1976). Buenos Aires: Fondo de Cultura Económica.

Foucault, M. (2008). Verdad y poder. Diálogo con M. Fontana. En: M. Focault, Un diálogo sobre el poder y otras conversaciones. Madrid: Alianza Editorial.

Foucault, M. (2002), Vigilar y Castigar. Nacimiento de la prisión. Buenos Aires: Siglo XXI Editores.

Gaby. (2016) Sobre Stop Gordofobia, La Gordura Masculina Y La Presión Estética Machista. Stop gordofobia. Recuperado de https://web.archive.org/web/20160430215159/http://www. stopgordofobia.com/sobre-stop-gordofobia-la-gorduramasculina-y-la-presi\%C3\%B3n-est\%C3\%A9tica-machista

Goffman, E. y Guinsberg, L. (1970). Estigma: la identidad deteriorada. Buenos Aires: Amorrortu.

Hall, S., Restrepo, E., Walsh, C. E. y Vich, V. (2014). Sin garantías: trayectorias y problemáticas en estudios culturales. Universidad del Cauca.

Harvey, D. (2007). Una breve historia del neoliberalismo. Oxford: Oxford University Press. 
Harjunen, H. (2016). Neoliberal bodies and the gendered fat body. Routledge.

Healy, K. (2001). Trabajo social: perspectivas contemporáneas. Ediciones Morata.

Lupton, D. (2013) Fat Politics: Collected Writings. Sydney: University of Sydney.

Matus, T. (2017). Punto de Fuga. Imágenes dialécticas de la crítica en el Trabajo Social Contemporáneo. Tomo 1. Buenos Aires: Editorial Espacio.

Matus, T. (2018). Transformación y Abismo: La pasión catastrófica del Trabajo Social. En: M. Flotts y B. Castro (eds.), Imaginarios de transformación: el trabajo social revisitado (pp. 21-46). Santiago de Chile: RIL Editores.

Moreno, M. L. (2015). Una historia de gordxs Breve análisis sobre los dispositivos de normalización de los cuerpxs. En: Jornadas "Discurso y poder: Foucault, las ciencias sociales y lo jurídico". Argentina: Universidad de Lanús.

Muñoz, G. (2018). Epistemologías críticas e intervención social. En: M. Flotts y B. Castro (eds.) Imaginarios de transformación: el trabajo social revisitado (pp. 137 - 160). Santiago de Chile: RIL Editores.

Muñoz, G. y Pantazis, C. (2019). Social exclusion, neoliberalism and resistance: The role of social workers in implementing social policies in Chile. Critical Social Policy, 39(1), 127-146. DOI: https://doi.org/10.1177/0261018318766509

National Eating Disorders Association. (2016). Factores de riesgo para los trastornos de la conducta alimentaria. Recuperado de: https://www.nationaleatingdisorders.org/factores-de-riesgo-para-los-trastornos-de-la-conducta-alimentaria

Navajas-Pertegás, N. (2017). La gordofobia es un problema del trabajo social. AZARBE, Revista Internacional De Trabajo Social $Y$ Bienestar, (6), 37-46. Recuperado de https://revistas.um.es/ azarbe/article/view/297181

Organización Mundial de la Salud. (16 de febrero de 2018). Obesidad y sobrepeso Recuperado de: https://www.who.int/es/newsroom/fact-sheets/detail/obesity-and-overweight

Portela de Santana, M. L., Da Costa Ribeiro Junior, H., Mora Giral, M. y Raich, R. M. (2012). La epidemiología y los factores de ries- 
go de los trastornos alimentarios en la adolescencia: una revisión. Nutrición hospitalaria, 27(2), 391-401. Recuperado de: http://scielo.isciii.es/scielo.php?script=sci_arttext\&pid $=$ S0212-16112012000200008

Rothblum, E. D. \& Solovay, S. (Eds.). (2009). The fat studies reader. NYU Press.

Sossa Rojas, A. (2011). Análisis desde Michel Foucault referentes al cuerpo, la belleza física y el consumo. Polis. Revista Latinoamericana, (28).

Toscano, D. G. (2008). El bio-poder en Michel Foucault. Universitas philosophica, 51, 39-57. Recuperado de: https://www.redalyc.org/pdf/4095/409534415003.pdf

Vargas, S. C. (2016). Construcción Social de la Gordura en niñas y niños de escolaridad primaria, residentes en Tijuana. Recuperado de: http://colef.repositorioinstitucional.mx/jspui/handle/1014/441

Vargas-Baldares, M. J. (2013). Trastornos de la conducta alimentaria. Revista Médica de Costa Rica y Centroamérica, 70(607), 475482. Recuperado de: https://www.medigraphic.com/pdfs/ revmedcoscen/rmc-2013/rmc133q.pdf

Wann, M. (2009). Fat studies: An invitation to revolution. In E. Rothblum \& S. Solovay (eds.), The fat studies reader (pp. xi-xxvi). New York: New York University Press.

Weinstock, J. \& Krehbiel, M. (2009). Fat youth as common targets for bullying. In E. Rothblum \& S. Solovay (eds.), The fat studies reader (pp. 120-126). New York: New York University Press. 